\title{
La libération des otages allemands au Liban : analyse politique et judiciaire (Partie 1)
}

Sylvie Lemasson

\section{OpenEdition}

1 Journals

\section{Édition électronique}

URL : http://journals.openedition.org/conflits/145

DOI : 10.4000/conflits. 145

ISSN : $1777-5345$

Éditeur :

CCLS - Centre d'études sur les conflits lilberté et sécurité, L'Harmattan

\section{Édition imprimée}

Date de publication : 21 mai 1992

ISSN : 1157-996X

\section{Référence électronique}

Sylvie Lemasson, "La libération des otages allemands au Liban : analyse politique et judiciaire (Partie 1) », Cultures \& Conflits [En ligne], 05 | printemps 1992, mis en ligne le 07 janvier 2003, consulté le 30 mars 2021. URL : http://journals.openedition.org/conflits/145; DOI : https://doi.org/10.4000/conflits. 145

Ce document a été généré automatiquement le 30 mars 2021.

Creative Commons License 


\title{
La libération des otages allemands au Liban : analyse politique et judiciaire (Partie 1)
}

\author{
Sylvie Lemasson
}

Contrairement à ses partenaires occidentaux français, britanniques et américains, la République fédérale d'Allemagne n'a pas d'antécédents de puissance mandataire ou coloniale au Proche-Orient et encore moins de prétention à un contrôle - ou à une hégémonie - politique régionale. Bien qu'étant la première force commerciale européenne au Machreq, elle ne cherche pas à convertir son capital économique en avantage politique, au risque d'ailleurs de perdre l'un sans posséder l'autre. Aussi la RFA ne sera-t-elle pas perçue par les différents groupes terroristes, qui sévissent à partir de la scène libanaise, ou encore par les états "parrains" pouvant exercer sur eux une influence autant stratégique que logistique, comme une cible privilégiée dans leurs relations avec les Occidentaux. L'objectif des preneurs d'otages libanais se résume globalement à une équation simple ${ }^{1}$ : miner la présence et la politique étrangère des puissances occidentales au Proche-Orient, en faisant pression sur elles. Il s'agit pour le mouvement Hezbollah d'obédience chiite iranienne, duquel proviennent les kidnappeurs comme les gardiens d'otages ${ }^{2}$, et pour l'Iran, qui commandite la capture des ressortissants de nations tenues pour "ennemies", d'amener les pays acteurs du théâtre israélo-arabe et arabo-persan à se retirer ou à modifier leurs alliances. La France, par exemple, aura à répondre à un marchandage précis sur les prises d'otages ${ }^{3}$. Les revendications du Hezbollah porteront essentiellement sur la libération de prisonniers chiites détenus dans la prison israélienne de Atlit et de ceux détenus au Koweït. Celles des Iraniens relèvent d'intérêts nationaux bien définis et sériés, dont le remboursement par la France de la dette d'"Eurodif" - et le versement d'intérêts considérables à l'Iran -, l'arrêt de la coopération militaro-commerciale avec l'Irak, l'expulsion d'opposants iraniens réfugiés en France et la libération de Anice Naccache ${ }^{4}$. I1 en va tout autrement pour la RFA sur ce damier proche-oriental. D'abord, elle n'a aucun contentieux à gérer avec les pays de la région. Elle est, de surcroît, le premier partenaire économique et l'"ami" politique occidental de l'Iran ${ }^{5}$. Enfin, et dans ces 
conditions, l'enlèvement de deux de ses ressortissants, en 1987, résulte d'une double singularité quant à son motif : le choix des "victimes" et le moment de leur enlèvement. En effet, comparé au nombre des otages occidentaux détenus prisonniers depuis 1984/85 - première grande vague d'offensive terroriste -, et à leur identité sociale ${ }^{6}$, le cas de Rudolf Cordes et de Alfred Schmidt se distingue de manière significative. Respectivement responsable commercial de la filiale libanaise de la compagnie chimique Hoechst et technicien de Siemens, ils apparaissent moins comme des pions sacrifiés des nations visées pour leur politique de puissance ou d'influence régionale que comme les représentants d'un pays impliqué malgré lui, du fait de ses décisions judiciaires, dans la tourmente terroriste. Tous deux ne sont pas choisis pour le symbolisme de leur fonction - diplomate, chercheur, enseignant, journaliste, militaire ou médiateur - qui marque l'emprise culturelle, sociale ou politique de pays comme la France, les Etats-Unis et la Grande-Bretagne. Ils ne sont pas non plus enlevés, le 17 janvier 1987 pour Cordes et le 20 du même mois pour Schmidt, en vue d'affaiblir une présence politique allemande, somme toute diffuse, et encore moins des relations commerciales qui ne sont aucunement remises en cause, mais en réponse à l'arrestation de Mohamed Hamade pour port d'explosifs liquides, à Francfort le 13 janvier 1987. L'enlèvement des Allemands est orchestré par le "parti de Dieu" libanais, dont un des chefs principaux est l'un des cinq frères Hamade, de confession chiite, Abdel Hadi. II s'opère sans lien temporel et structurel avec les engagements régionaux du pays d'origine des otages à l'inverse des autres prises d'otages. Ces dernières s'inscrivent dans des contextes précis ou dans une logique de pression déterminée à l'encontre de politiques gouvernementales, dénoncées par les ravisseurs ou par les états qui dirigent leurs actions.

Les membres actifs du Hezbollah décident d'enlever des citoyens allemands - mais sans porter atteinte aux représentations diplomatico-politiques de la RFA comme il l'a été envisagé - afin d'obliger Bonn à ne pas extrader Mohamed Hamade vers les USA. Washington réclame, en effet, le jeune Libanais pour le juger sous quatre chefs d'inculpations : le détournement, sur l'aéroport de Beyrouth en juin 1985, du Boeing 727 de la compagnie TWA, le meurtre, au cours de cette opération, d'un passager de nationalité américaine, Robert Stethem, prises d'otages et, enfin, coups et blessures. Si elle n'obtempère pas aux injonctions des terroristes, la RFA est alors à même d'être exposée à des représailles, en sa qualité d'alliée politique de la puissance "impérialiste" d'outre-Atlantique. Lorsque Abbas Hamade, l'un des frères de Mohamed sera à son tour arrêté sur le territoire de la République fédérale, le 26 janvier 1987, les revendications du Hezbollah. S'élargiront à la libération des deux Libanais pour toute remise en liberté des ressortissants allemands.

Face à ce double chantage dans ce qui devient "l'affaire Hamade", et qui porte avant tout sur un échange humain et non sur les engagements et les orientations d'une politique extérieure, la RFA doit, néanmoins, gérer un certains nombre de contraintes inhérentes à sa politique intérieure et étrangère. Diverses questions se posent. Notamment, comment le processus décisionnel allemand accommodera-t-il logique politique et judiciaire ? Comment l'état de droit et démocratique allemand préserve-t-il sa légitimité et sa souveraineté dans le processus de négociations comme dans le traitement judiciaire de 1'"affaire Hamade" ? Surtout lorsque cette dernière se trouve intimement liée au développement du réseau terroriste Ali Fouad Saleh, organisant des actes terroristes en France, et lorsqu'elle doit répondre aux demandes d'extradition américaines. 
Contraintes internationales

Pour la libération de ses ressortissants, la RFA doit agir en tenant compte de tensions externes qui s'articulent autour de trois pôles et qui pèsent de manière contraire sur la conduite de la politique allemande: les requêtes américaines, les revendications des ravisseurs et la recherche d'une politique de coordination tant européenne qu'occidentale dans le refus de négocier, donc de céder, aux preneurs d'otages.

Les USA

Lorsque le 13 janvier 1987 Mohamed Ali Hamade, de nationalité libanaise, est interpellé sur le territoire ouest-allemand en possession de neuf litres de nitrate de méthyle, les recherches révèlent qu'il est sous le coup d'un mandat d'arrêt international lancé par les Etats-Unis. Ces derniers demandent immédiatement aux autorités de Bonn l'extradition du jeune terroriste, eu égard à l'accord germano-américain d'assistance juridique, conclu le 20 juin 1978. Mais l'enlèvement le 17 janvier de R. Cordes et de A. Schmidt le 20 janvier, puis l'arrestation de Abbas Hamade le 26 janvier à l'aéroport de Francfort, en possession également d'explosifs liquides à son retour de Beyrouth, compliquent singulièrement le quasi-automatisme d'une telle procédure. Le sort des otages allemands dépend désormais, selon leurs ravisseurs, de la libération des frères Hamade. Le gouvernement de Bonn se trouve face à un dilemme. Pour satisfaire ses alliés américains, extrader Mohamed Hamade revient à perdre toute influence sur le déroulement des événements du procès aux Etats-Unis. C'est refuser de se plier aux mises en garde du "clan" et de prendre le risque de provoquer les membres du Hezbollah. A l'inverse, si le gouvernement accepte de traduire en justice le cadet des Hamade en RFA, il ménage en partie les exigences de la famille libanaise mais aux dépens de ses relations ou de sa solidarité avec Washington. Finalement le 25 juin 1987, Bonn décide de ne pas extrader Mohamed Hamade et de le soumettre aux tribunaux allemands ${ }^{7}$. Le gouvernement s'octroie ainsi une marge de man cuvre minimale pour "négocier" avec les ravisseurs. Le traitement politico-juridique du cas de Mohamed Hamade sera vivement critiqué par les autorités américaines. Et cela tant en raison du refus allemand de procéder à l'extradition que de la clémence juridictionnelle de la cour de Frankfort. Washington reproche en effet aux juges du Land de Hesse leur conclusion prise en avril 1988. Elle s'appuie sur le fait que le jeune Libanais n'était pas encore âgé de 21 ans lors du détournement et du meurtre commis à Beyrouth en juin 1985 pour décider de le faire comparaître devant un tribunal pour mineurs. Dans ce cas, non seulement celui-ci échappe à la peine capitale qu'il encourait aux Etats-Unis - et qui n'existe pas en RFA - mais il est passible d'une peine de dix ans d'emprisonnement maximum - et échappe de la sorte à une sentence qui relève normalement de la perpétuité devant les cours d'assises allemandes ${ }^{8}$. En revanche lors du procès concernant Abbas Hamade, les Américains exprimeront leur satisfaction.

Agé de 29 ans, il possède la double nationalité libanaise et allemande, depuis $1984^{9}$.

Domicilié en RFA, dans le Land de la Sarre, il relève directement d'une juridiction allemande, celle de Düsseldorf, devant laquelle il sera accusé de trois délits : complicité d'enlèvements ${ }^{10}$, chantage à l'encontre de l'état allemand - en se servant des menaces pesant sur la vie des deux otages pour éviter que Mohamed ne soit extradé -, détention de matière explosive. Le 19 avril 1988, après quatre mois de procès (celui-ci débuta le 5 janvier), Abbas Hamade est condamné à treize ans de prison, et reconnu coupable de ces trois chefs d'inculpation. Les avocats de la défense avaient demandé une peine de un an maximum pour la détention illégale d'explosifs. Le procureur général avait 
requis une peine de onze ans et demi. Le département d'état américain se félicite donc de la sévérité de la sentence qui est supérieure à la demande du procureur et, par la voix de Phyllis Oakley, qualifie la décision du tribunal de Düsseldorf de "message clair aux terroristes".

Pourtant, le message n'est pas si clair puisque le président de la cour de Düsseldorf, Klaus Arendt, rappelle aux ravisseurs du dernier otage allemand, Rudolf Cordes, dont il

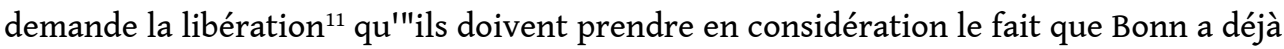
cédé à leurs pressions en n'extradant pas Mohamed Hamade". Du fait de son contexte, la décision de Düsseldorf souligne deux points qui paraissent en contradiction. D'une part l'"aveu" d'une concession venant des juges, d'autre part la sévérité de la peine prononcée, qui ne semble pas tenir compte du chantage de la famille Hamade et vise à proclamer la pleine indépendance de l'autorité judiciaire dans le jugement de Abbas. En outre, en saluant la fermeté des magistrats, les autorités gouvernementales soulignent leur refus de "brader" plus avant la libération du dernier otage allemand; après avoir déjà répondu positivement aux attentes du Hezbollah concernant le cas de Mohamed.

Les propos de Klaus Arendt peuvent, cependant, ne pas contredire l'impression de fermeté, si l'on comprend que Bonn se donne les moyens d'une véritable liberté d'action ou au moins une marge de manoeuvre politique en apportant des "garanties suffisantes" aux complices des frères Hamade quant au sort "réservé" à ces derniers en RFA, en leur faisant admettre qu'il faut un certain temps pour que la décision judiciaire puisse faire l'objet d'une atténuation acceptable aux yeux de tous (opinion publique allemande, USA).

Le Hezbollah et le clan Hamade au Liban

Les exigences des ravisseurs sont clairement exprimées dès le départ. Une de leurs deux conditions pour la libération des otages était la non-extradition de Mohamed Hamade. Ils l'obtiennent, le 24 juin 1987, par le gouvernement de Bonn. Le 7 septembre 1987, Alfred Schmidt est remis en liberté par ses geôliers. La seconde des conditions se rapportait à la libération elle-même des frères Hamade. Le Hezbollah ne l'obtient pas. Quand prend fn le procès de Abbas Hamade et lorsque commence celui de son jeune frère Mohamed, le 5 juillet 1988, le "parti de Dieu" détient encore Rudolf Cordes, son unique moyen de pression, bien que d'autres prises d'otages ne soient pas exclues, si nécessaire.

De quelles assurances disposaient les ravisseurs, de la part de Bonn à l'automne 1987, pour prendre en toute confiance une première initiative? A moins qu'en faisant montre de leur bonne disposition, de manière unilatérale, ils aient voulu influer sur la procédure judiciaire des deux Libanais? Quoiqu'il en soit marchandage concerté ou non -, leur décision était destinée à lier celle de leur otage au sort de Mohamed et Abbas Hamade en RFA. La libération rapide d'Alfred Schmidt peut logiquement répondre au geste politique et juridique allemand en faveur du premier des frères (la nonextradition vers les USA). Celle de Rudolf Cordes, le 12 septembre 1988, qui s'opère après le procès de Abbas et au cours de celui de Mohamed Hamade, dépend, selon toute vraisemblance, d'une négociation plus large. Les termes de cette dernière peuvent relever de trois domaines, jouant chacun un rôle déterminé pour la conduite de tractations : économique, juridique et politique. Les ravisseurs doivent se prêter à une négociation compte tenu du fait que la RFA, état de droit, ne peut transiger sur le principe de sa légitimité ou sur toute revendication qui affaiblirait sa souveraineté. De plus, ses intérêts comme ses desseins politiques, qui apparaissent modérés sur la scène 
proche-orienta1e, sont la source de peu de conflits et donc l'objet de "marchandages" restreints. Les termes du contrat entre les membres du Hezbollah et la République fédérale peuvent reposer avant tout sur le terrain commercialo-financier, fer de lance de la présence allemande dans la région mais également de la force et de l'identité allemandes en général. L'hypothèse d'une rançon, qui monnayerait le "bon vouloir" des ravisseurs, doit être retenue tout comme celle de promesses relatives à des contrats ou arrangements commerciaux dont bénéficieraient des pays tiers, impliqués directement ou indirectement dans les prises d'otages. Cette composante matérielle, inhérente aux clauses d'une négociation, sera vérifiée en seconde partie de cette étude, dans le cadre des différentes réponses allemandes apportées aux ravisseurs et aux états responsables des prises d'otages.

Deux autres hypothèses, qui touchent au rôle du judiciaire et du politique en RFA, permettent d'avancer l'idée que les autorités fédérales assurent les ravisseurs d'engagements allemands spécifiques à moyen terme - le facteur temps demeurant décisif pour toute crédibilité accordée à l'une ou l'autre partie engagée dans les discussions. Par exemple: l'éventuel engagement de Bonn d'"alléger" les peines des frères Hamade, dans un délai suffisamment raisonnable pour les autorités gouvernementales allemandes et suffisamment honorable pour les terroristes, pourrait expliquer que les ravisseurs libèrent Rudolf Cordes, le 12 septembre 1988, alors que le procès de Mohamed Hamade n'est pas parvenu à son terme.

Toutefois, cette opération est possible uniquement grâce au soutien des pays qui exercent une influence déterminante sur les structures du Hezbollah et sur les différents groupes qui le composent : l'Iran et la Syrie. L'Iran, pour son rôle de "tuteur" vis-à-vis du Hezbollah en tirant les ficelles de ses organisations clandestines comme le Jihad islamique ; la Syrie, en raison du contrôle stratégique qu'elle exerce dans la zone géographique où évolue le mouvement chiite, principalement à la périphérie sud de Beyrouth et à Baalbek. Ainsi, bien que le sort des otages ne dépende pas directement de Damas, aucun accord ne peut se réaliser sans son assentiment du fait de son "omniscience" au Liban ${ }^{12}$. Ces états "commanditaires", par la voix du gouvernement iranien et du président Hafez el Assad, doivent assurer eux-mêmes aux ravisseurs que certaines "garanties" ont été trouvées pour une "solution du problème des frères Hamade ${ }^{13}$.

En contrepartie, ils font pression sur les ravisseurs pour que ces derniers libèrent avant le procès leur dernier otage. Mais ces services se paient. Ils sont liés, en toute logique, à l'attitude politique de la RFA au Proche-Orient et plus précisément au traitement des affaires proche-orientales au sein de la Communauté européenne où Bonn joue un rôle non négligeable.

Il s'agira pour les responsables des prises d'otages de conduire l'Allemagne à se faire l'interprète d'intérêt(s) particulier(s) de Damas et de Téhéran ou de plaider leur réhabilitation auprès de ses partenaires de la $\mathrm{CE}$. A partir de ces attentes, sinon conditions, le gouvernement fédéral aura alors à gérer un nouveau dilemme. Comment répondre à ses intérêts nationaux compatibles au demeurant avec ceux des Iraniens et des Syriens sans développer une politique de cavalier seul qui fasse défaut à tout esprit de solidarité en matière de lutte antiterroriste ou de politique européenne commune? Le Cheikh Fadlallah, chef religieux des chiites libanais et du mouvement Hezbollah, en appelant la RFA, qui "est un état souverain" à "prendre en compte ses propres intérêts" joue sur ce dilemme. Pour lui, "les Allemands sont (suffisamment) pragmatiques et 
raisonnables" pour faire passer leurs intérêts nationaux avant les discours de solidarité occidentale ${ }^{14}$

La Communauté européenne

Bien que la règle d'or des pays européens, et des Etats-Unis soit de refuser toute tractation avec les terroristes, il y a des libérations plus rapides que d'autres, dont celles concernant la RFA, qui ne peuvent faire douter de l'existence de conditions particulières.

Il est également vrai que la remise en liberté des otages occidentaux au Liban qui s'opérera progressivement, pour la majorité d'entre eux, à l'issue de la guerre du Golfe en $1991^{15}$, se fera, de manière générale, aux termes de négociations inavouées ou de marchandages inavouables ${ }^{16}$.

A l'égard de la Syrie et de l'Iran, la République fédérale a adopté une politique, sinon flexible, du moins modérée, contrairement à ses partenaires de la Communauté européenne. Ainsi en 1988 lors de la présidence de la CE par la RFA, son ministre des Affaires étrangères, Hans-Dietrich Genscher, est le seul représentant européen à engager une tournée dans les principales capitales du Moyen-Orient. II se rendra notamment à Damas, en janvier 1988, pour relancer le dialogue euro-arabe en demandant à ses partenaires de Bruxelles la levée des sanctions appliquées par la CEE contre la Syrie. Ces sanctions, avant tout économiques, avaient été proposées par la Grande-Bretagne et décidées après les procès des frères Hindawi, à la fin de l'année $1986^{17}$. Le régime de Hafez el Assad est alors accusé par les Douze de soutenir le terrorisme international. Les services secrets syriens seront d'ailleurs mis en cause par un tribunal allemand qui juge l'un des frères du "clan" Hindawi, impliqué dans un attentat à la bombe à Berlin-ouest en mars 1986. Alors que Londres rompt ses relations diplomatiques avec Damas et que la CEE dans son ensemble opte pour un arrêt de toute aide communautaire à la Syrie, Bonn trouve un compromis sur le plan bilatéral. Soumise à des pressions contraires, d'un côté britannique et américaine pour la fermeté, de l'autre syrienne et arabe comme autant de mises en garde, la RFA campe sur une position pragmatique :

- rappel de son ambassadeur à Damas avant l'expiration de son mandat, accompagné de l'expulsion de quatre membres de l'ambassade syrienne à Bonn ;

- demande à Damas d'une condamnation verbale du terrorisme international - dirigée en fait contre ses propres services secrets ${ }^{18}$. L'enjeu d'une normalisation au plan bilatéral est d'importance pour Bonn mais l'exercice de réhabilitation non moins délicat au sein de la $\mathrm{CE}^{19}$. En 1988, Hans-Dietrich Genscher cherche à absoudre Damas de toute influence sur les affaires du terrorisme tout en espérant que cette influence puisse, néanmoins, s'exercer sur les détenteurs de son ressortissant, Rudolf Cordes. A l'égard de l'Iran, la RFA se démarque de ses partenaires européens. Hans-Dietrich Genscher sera le premier ministre des Affaires étrangères de la Communauté à se rendre à Téhéran, en juillet 1984. Il se fera l'interprète des intérêts iraniens auprès de la CE en estimant que "rien ne serait plus erroné que d'isoler ce grand et cet important pays ${ }^{20}$. En 1987, le ministre des Affaires étrangères iranien, Ali Akbar Velayati, se rend à Bonn et Hans Dietrich Genscher à nouveau à Téhéran en novembre 1988. La réception du ministre iranien, en juillet 1987, suscite l'irritation de Paris qui traverse une crise aiguë avec la République islamique. Les relations diplomatiques entre la France et l'Iran 
sont suspendues. Londres décide de geler les siennes avec ce dernier et son ministre des Affaires étrangères, Sir Geoffrey Howe, propose, en septembre 1987 à l'ONU, un embargo sur les armes à destination de Téhéran. Dans le même temps, il ordonne la fermeture du bureau d'achat d'armements de l'Iran à Londres ${ }^{21}$.

De son côté, Bonn recueille les fruits de ses "bonnes" relations entretenues avec les gouvernements syriens et iraniens pour négocier la libération de ses otages. La solidarité européenne en matière de lutte contre le terrorisme ou de politique étrangère au Moyen-Orient doit subir de lourds accrocs que provoque la force des intérêts nationaux. La RFA en accentue les effets par son refus, en 1988, de créer une unité antiterroriste, susceptible de participer à l'échelon international à la lutte contre la piraterie de l'air. Bonn se verra reprocher de faire trop de concessions aux ravisseurs alors qu'elle-même adressait des critiques similaires à Paris, avant d'être touchée à son tour par les prises d'otages.

Les contraintes internes

Le processus décisionnel, qui conduit à la libération des otages allemands, résulte d'une dynamique duelle: d'un côté, l'interaction entre la politique internationale et nationale, de l'autre, l'interférence entre le pouvoir politique et le pouvoir judiciaire. Les réponses de Bonn aux membres du Hezbollah dépendent de ces différents niveaux d'interpénétration tout en ayant comme objectif central d'obtenir la libération de ses ressortissants sans donner l'impression de se soumettre au chantage des ravisseurs. Il en va, en effet, de la crédibilité et de l'autorité politique de la République fédérale comme de la souveraineté et de l'intégrité de son appareil judiciaire.

$\mathrm{Au}$ plan interne, la politique allemande se trouve confrontée à plusieurs défis. Tout d'abord, elle doit adopter une attitude appropriée aux choix précédemment opérés à l'égard de la RAF (Rote Armee Fraktion). Une cohérence s'impose entre l'inflexibilité du gouvernement fédéral manifestée lors de l'affaire Schleyer et la rigueur recherchée dans celle des otages du Liban. Ensuite pour être efficace, le processus décisionnel doit reposer sur un certain nombre d'éléments nécessaires à sa cohérence : unité de lieu et de temps dans les prises de décision; médiateurs désignés par Bonn et reconnus comme tels par les organisateurs ou les commanditaires des enlèvements ; repérage et connaissance exacts de l'identité de ces derniers.

Enfin, les responsables politiques, en charge du règlement du problème des otages doivent, aussi, et surtout, prendre en considération la logique et le mécanisme du pouvoir judiciaire. L'indépendance de celui-ci peut, par la sévérité de ses verdicts, contrecarrer les engagements politiques contractés auprès des Ravisseurs ${ }^{22}$.

\section{NOTES}

1. Et cela contrairement à la stratégie des attentats délibérément commis sur un territoire national donné. Voir l'Etude de Didier Bigo, Les attentats de 1986 en France : un cas de violence transnationale et ses implications, préparée pour la présentation au 
XVème congrès mondial de l'Association internationale de science politique, Buenos Aires, 1991, pp. 17-19.

2. Les différents groupes, Jihad islamique, OJR, JILP qui revendiquent attentats ou prises d'otages, entre 1985 et 1991, sont tous reliés au "parti de Dieu", le Hezbollah, créé en 1982 sur le modèle iranien et placé sous le contrôle du ministre de l'Intérieur de Téhéran, Ali Akbar Mohtachemi. Puis, le mouvement libanais abritera une série de factions qui vont $s$ affronter pour le pouvoir en Iran et des clans chiites divers, proches des services syriens ou de groupes palestiniens.

3. Un marchandage différent de celui concernant la cessation des attentats organisés par l'organisation de Ali Fouad Saleh à Paris.

4. Il dirige un commando qui tente d'assassiner à Paris, en 1980, Chapour Baktiar, ancien Premier ministre du Shah.

5. La RFA entretient des contacts réguliers avec Téhéran. Pendant près de trente ans, l'Iran reste son premier débouché industriel et l'un de ses trois principaux fournisseurs de pétrole nu Moyen-Orient. Au milieu des années 80, alors que les relations entre Téhéran et les autres capitales occidentales n'ont cessE de se dégrader à partir de 1979, la République fédérale tire profit du vide laissé par le départ américain ou le recul britannique et français. Elle renforce sa place de premier fournisseur occidental du régime islamiste. Au début de la guerre du Golfe, entre l'Iran et l'Irak, la RFA se démarque de l'attitude ferme de ses partenaires, alors que le régime iranien est dénoncé et que l'Irak apparait comme un rempart à l'extension de l'islamisme chiite. Bonn se montrera réticente à suivre les sanctions économiques préconisées et appliquées par les Etats-Unis à l'encontre de l'Iran. Durant la guerre, la diplomatie allemande se singularise dans la "quarantaine" que subit le régime de Khomeyni. Bonn maintient ses relations diplomatiques avec Téhéran et son ministre des Affaires étrangères sera le premier ministre de l'OTAN - excepté celui de la 7tirquie - à se rendre en Iran en juillet 1984. D'autres visites officielles suivront par la suite, en 1987 et 1988. En 1987, la RFA exporte pour 1,6 milliard de dollars en Iran, loin devant la Grande-Bretagne avec 503 millions, la France, 179 et les USA, 54.

6. Pour plus de détails, voir annexe II.

7. Bulletin Presse und informationsamt der Bundesregierung, $n^{\circ}$ 65, 30/06/87, p. 572.

8. Le 16 janvier 1987, le porte-parole du ministre de la Justice allemand évoquait lu possibilité d'une extraditio à la condition que les USA renoncent à la peine capitale 9. Marié à une Allemande en 1980, Marin Susanna Müller, il divorce en 1986 et vit avec Mouna Hage, fille de Rachid Hage, un Libanais poursuivi en RFA pour détention illégale d'explosifs dans le cadre de l'affaire Hamade. Après l'arrestation de Abbas, les enquêteurs allemands retrouveront près de la frontière française, à Becingen, une cache d'explosifs identiques à ceux transportés par les deux frères et à ceux utilisés dans plusieurs attentats à Paris.

10. Voir organigramme. Au cours du procès de Abbas Hamade, le tribunal de Düsseldorf réunira à raison de deux fois par semaine et entendra près de 70 témoins dont l'ancien otage Alfred Schmidt.

11. Dès l'ouverture de son procès, Abbas Hamade a appelé de lui-même son "clan" à libérer Rudolf Cordes. Il reconnaît ainsi implicitement sa complicité avec les ravisseurs en prétendant influer sur le cours de la détention du ressortissant allemand.

12. Cf. José Garçon, "Les chemins d'un dénouement", in Libération, 5.5.88.

13. Comme l'indique le Hezbollah dans un communiqué à la suite de la libération de Rudolf Cordes, Cf. Le Monde, 14.09.88. 
14. Interview in Der Spiegel, 36/ 1991, p.217.

15. La guerre du Golfe modifie les rapports de force inter et intra-régionaux comme les relations entre les principaux pays du Moyen-Orient et le monde occidental. La Syrie et l'Iran, qui exercent une influence sur les groupes du Hezbollah, trouvent intérêts, pour des raisons différentes mais convergentes, à traiter du sort des otages et à provoquer leur libération.

16. Cf. Marc Kravetz, "Otages, sept ans après, la page se tourne", in Libération, 05.12.91. 17. Nezar Hewat Hindawi sera notamment condamné à 45 ans de prison pour avoir mené un attentat à la bombe contre un avion israélien de la compagnie El Al.

18. Bien que les services secrets syriens soient accusés par les Occidentaux principalement par les Américains et les Britanniques - d'avoir concouru à la préparation de l'attentat du 29 mars 198G, contre le centre germano-arabe de BerlinOuest, pour lequel Ahmerl Hasi et Farouh Salameh sont condamnés à 14 et 13 ans de prison la RFA pense - ou préfère penser - que Damas ne contrôle pas ses services secrets. C'est pourquoi, Bonn invite Damas à surveiller de plus près ses "agents" tout en lui suggérant de participer à la lutte anti-terroriste. La réaction allemande est d'autant plus étonnante et l'exercice d'absolution de Bonn délicat que Hasi est également soupçonné d'avoir participé à l'attentat perpétré contre la discothèque Berlinoise "La belle", entraînant la mort de trois personnes et en blessant plus de 200 autres.

19. Le ministre des affaires étrangères a également besoin de l'appui de la Syrie pour mener à bien la mission qu'il se fixe. Celle d'oeuvrer en faveur de la paix dans le Golfe, entre l'Irak et l'Iran, et de l'application de la résolution 598 de l'ONU. L'une des conditions du succès de d'initiative de Genscher est de conduire Bagdad à davantage d'ouverture, voire de souplesse, avec l'appui des autres pays influents arabes, dont la Syrie.

20. Süddeutsche Zeitung, 13.07.84, Volksblatt, 13.07.84, Le Figaro, 23.07.84 et Le Monde, 25.07.84.

21. Le " bureau d'approvisionnement militaire" de la capitale britannique constituait depuis 1981 une importante plaque-tournante pour l'achat de matériels destinés à l'armée de terre, à l'aviation el à la marine de guerre iraniennes. Celte décision du Foreign Office est précipitée par l'attaque, le 21 septembre 1987, du pétrolier britanniqueb Gentle Breeze par une une dette iranienne.

22. Le juge Müchenberger, président de la cour de Francfort se déclare d'ailleurs soulagé à l'annonce de la libération de Rudolf Cordes : . () Les constantes spéculations sur l'influence qu'aurait pu avoir le sort de Rudolf Cordes sur le progrès de Mohamed Hamade vont cesser () Pour le tribunal, il a toujours été question de conduire cette procédure de façon indépendante et en respect des lois en vigueur. Nous n'avons pas à faire de diplomatie dans ces lieux", Libération, 14.09.88

\section{RÉSUMÉS}

L'enlèvement de deux citoyens allemands au Liban en 198î puis de quatre autres en 1989, relève d'une problématique singulière, différente de celle concernant les pays occidentaux victimes 
également de prises d'otages. Le mouvement du Hezbollah demeure certes l'auteur principal de toutes ces actions terroristes mais, dans le cas de la RFA, il s'agit moins pour lui de porter atteinte à la présence allemande au Moyen-Orient que de chercher à exercer un chantage humain sur les autorités politiques de Bonn afin qu'elles libèrent Mohamed et Abbas Hamade, arrêtés à Francfort en possession d'explosifs. Aussi la singularité des motifs qui commandent l'enlèvement de citoyens de la République fédérale met-elle en relief la spécificité de la diplomatie allemande dans la région. En reposant sur des intérêts économiques et des particularismes historiques bien définis, elle bénéfice d'un certain nombre d'atouts politiques pour prétendre à des négociations rapides.

The kidnapping of two German citizens in Lebanon in 1987, followed by that of four more in 1989 , have their own specificity which differs from the taking of many other Western hostages. While Hezbollah undoubtedly is the main force behind these terrorist acts, in the case of the Federal Republic, it is not so much a question of inflicting damage on German interests in the Middle East, as of blackmailing Bonn authorities into releasing Mohamed and Abbas Hamade, arrested in Frankfurt in possession of explosives. Thus the motivations behind the kidnapping of citizens of the German Federal Republic reflect the role of German diplomacy in the region. Economic interests and specific historical factors provide the setting supposed to facilitate rapid negotiations.

\section{INDEX}

Index géographique : Liban

Mots-clés : coopération internationale, gestion de crise

Thèmes : Hezbollah

\section{AUTEUR}

\section{SYLVIE LEMASSON}

Chercheur associé au CERI 